\title{
Collective Dipole Bremsstrahlung in Fusion Reactions
}

\author{
V. $\operatorname{Baran}^{a b)}$, D.M. Brink ${ }^{c}$, M. Colonna ${ }^{a)}$, M. Di Toro ${ }^{a)}$ \\ a) Laboratori Nazionali del Sud, Via S. Sofia 44, I-95123 Catania, Italy and University of Catania, \\ b) NIPNE-HH, Bucharest, Romania, ${ }^{c)}$ Oxford University, U.K.
}

(December 1, 2018)

We estimate the dipole radiation emitted in fusion processes. We show that a classical bremsstrahlung approach can account for both the preequilibrium and the thermal photon emission. We give an absolute evaluation of the preequilibrium component due to the charge asymmetry in the entrance channel and we study the energy and mass dependence in order to optimize the observation. This dynamical dipole radiation could be a relevant cooling mechanism in the fusion path. We stress the interest in experiments with the new available radioactive beams.

PACS numbers: 24.30.Cz; 25.70.Jj; 25.70.Lm; 25.60.Pj

In the early stage of a heavy ion collision large amplitude collective motions can be excited. Monopole, dipole or quadrupole modes are typical examples. Tracing information from their decay provides the opportunity to learn about the features of fermionic systems in particular, extreme conditions.

If the colliding nuclei have different $N / Z$ ratios the charge equilibration process takes place. Several works have suggested that the related neutron-proton collective motion has the features of a Giant Dipole Resonance $(G D R)$ mode [1] 7]. In a microscopic approach based on semiclassical $B N V$ transport equations [8], it has been studied in detail how a collective $G D R$ responsenbdevelops in the entrance channel. In connection to this analysis three main phases can be identified. First, during the approaching phase the two partners, overcoming the Coulomb barrier, still keep their own response. Then it follows a dinuclear phase when the conversion of relative motion energy in thermal motion starts to take place, mainly due to nucleon exchange. The composite system is not thermally equilibrated and manifests, as a whole, a large amplitude dipole collective motion. The charge equilibration has initiated. Finally, during the third phase, the Compound Nucleus (CN) formation, the nucleus is thermally equilibrated. In a phonon approach [9, 10] it was shown [5] for the first time that an enhancement of the $G D R$ gamma emission should be observed if the number of $G D R$ phonons, $n_{G D R}^{(0)}$, when the $C N$ is formed, is larger than the value corresponding to a statistical equilibrium of the GDR (as oscillator) with the $C N$ (as heat bath). This effect has been experimentally evidenced in several fusion reactions 111 14].

However it is important to notice that also in the second (dinuclear) phase a $G D R$ is excited on top of nonequilibrium states 15 (Brink-Axel hypothesis). The number of $G D R$ phonons is even larger than $n_{G D R}^{(0)}$ [8] and consequently some pre-equilibrium $G D R$ photons can be emitted also at this stage. In the attempt to estimate this contribution we cannot use straightforwardly the "phonon" approach [5,9] which is based on the assumption of the existence of a thermally equilibrated nucleus. A way to face this problem is to directly apply a bremsstrahlung ("bremss") approach. Considering the evolution of the collective dipole acceleration from the time when it suddenly rises, at the beginning of the second phase, until it is completely damped to a pure "thermal" component, we can consistently calculate the whole contribution of the pre-equilibrium $G D R$ to the photon yield. This is the aim of our letter.

The total photon emission probability from the dipole mode oscillations, as given by the bremsstrahlung formula, can be expressed as [16] $\left(E_{\gamma}=\hbar \omega\right)$ :

$$
\frac{d P}{d E_{\gamma}}=\frac{2 e^{2}}{3 \pi \hbar c^{3} E_{\gamma}}\left(\frac{N Z}{A}\right)^{2}\left|X^{\prime \prime}(\omega)\right|^{2},
$$

where $X^{\prime \prime}(\omega)$ is the Fourier transform of the acceleration $X^{\prime \prime}(t)$ associated with the distance between the centers of mass of protons $(P)$ and neutrons $(N), X=R_{p}-R_{n}$. $A=N+Z$ is the system mass. Thus following the time evolution of the dipole mode along the fusion dynamics it is possible to evaluate, in absolute values, the corresponding pre-equilibrium photon emission. However it is interesting to notice that the "bremss" approach 17 actually provides a unified picture to account for the photon emission from both pre-equilibrium and thermal $G D R$. In fact Eq.(1) also allows to recover the usual expression for the $\gamma$-decay rate from a $G D R$ in thermal equilibrium with a $C N$ at temperature $T$. In this case the dipole mode will manifest thermal oscillations that by the fluctuation-dissipation theorem are completely determined only by its dissipative properties [18]. We may consider equivalently that these are the consequence of the action of a fictious, time dependent, random force $F(t)$ 19. Then the Fourier transform of the distance $X(t)$ can be expressed as: $X(\omega)=\alpha(\omega) \times F(\omega)$, where

$$
\alpha(\omega)=\alpha_{r}(\omega)+i \alpha_{i}(\omega)=\frac{1}{M_{\text {coll }}\left(\omega_{0}^{2}-\omega^{2}-i \frac{\Gamma}{\hbar} \omega\right)},
$$

is the response function of the dipole mode [21]. $M_{\text {coll }} \equiv$ $\frac{N Z}{A} m$ with $m=935 \mathrm{MeV}$ (nucleon mass) represents the collective mass of the neutron-proton relative motion and $\Gamma$ is the decay width.

The spectral density of mean square fluctuation of random force $<|F(\omega)|^{2}>$ is related to the properties of the 
system through the fluctuation-dissipation theorem, as it follows [18, 19]:

$$
<|F(\omega)|^{2}>=\frac{\hbar \alpha_{i}(\omega)}{|\alpha(\omega)|^{2}} \operatorname{cth}\left(\frac{\hbar \omega}{2 T}\right)=M_{\text {coll }} \omega \Gamma \operatorname{cth}\left(\frac{\hbar \omega}{2 T}\right) .
$$

Using the Parseval theorem and the ergodic hypothesis we have, for times $T_{0}$ very large, $\lim _{T_{0} \rightarrow \infty} T_{0}<$ $|F(\omega)|^{2}>=|F(\omega)|^{2}$. So, by introducing in Eq.(1) the Fourier transform $X^{\prime \prime}(\omega)$ of the acceleration, since $\left|X^{\prime \prime}(\omega)\right|^{2}=\omega^{4}|X(\omega)|^{2}$, we get the average photon emission probability per unit time:

$$
\lim _{T_{0} \rightarrow \infty} \frac{1}{T_{0}} \frac{d P}{d E_{\gamma}}=\frac{2}{3 \pi} \frac{e^{2}}{\hbar m c^{3}} \frac{N Z}{A} \frac{\Gamma E_{\gamma}^{4}}{\left(E_{\gamma}^{2}-E_{0}^{2}\right)^{2}+\Gamma^{2} E_{\gamma}^{2}} \operatorname{cth}\left(\frac{\hbar \omega}{2 T}\right)
$$

If we exclude the zero-point motion contribution and expand the hyperbolic function for $\hbar \omega>T$ we obtain:

$$
\begin{gathered}
\lim _{T_{0} \rightarrow \infty} \frac{1}{T_{0}} \frac{d P}{d E_{\gamma}}=\frac{1}{\hbar}\left(\frac{E_{\gamma}}{\pi \hbar c}\right)^{2} \\
\frac{1}{3} \frac{4 \pi e^{2} \hbar}{m c} \frac{N Z}{A} \frac{\Gamma E_{\gamma}^{2}}{\left(E_{\gamma}^{2}-E_{0}^{2}\right)^{2}+\Gamma^{2} E_{\gamma}^{2}} \exp \left(-\frac{E_{\gamma}}{T}\right)= \\
\frac{1}{\hbar}\left(\frac{E_{\gamma}}{\pi \hbar c}\right)^{2} \frac{\sigma_{a b s}}{3} \exp \left(-\frac{E_{\gamma}}{T}\right),
\end{gathered}
$$

where $\sigma_{a b s}$ is the $\gamma$-absorption cross section in the $G D R$ region. This is the well known formula for the gamma emission rate from hot $G D R$ [20]. It was previously obtained by using the detailed balance principle or from considerations on the statistical equilibrium between dipole mode and black-body radiation 21 .

Now we focus on the dynamical dipole mode in the entrance channel and we study the dependence of the pre-equilibrium dipole oscillations on the incident beam energy. We look at the relative weight vs. the statistical contribution.

In the following we present results concerning the charge equilibration process for the systems ${ }^{40} \mathrm{Ca}$ $(N / Z=1)$ on ${ }^{100} \mathrm{Mo}(N / Z=1.38)$, at $4 A M e V$ and ${ }^{16} \mathrm{O}(N / Z=1)$ on ${ }^{98} \mathrm{Mo}(N / Z=1.33)$ at various beam energies $(4,8,14$ and $20 A M e V)$. Our fusion dynamics is obtained in the framework of an accurate $B N V$ transport simulation of central collisions, see [8] for a detailed analysis.

The time evolution of the dipole moment $D(t)=$ $\frac{N Z}{A} X(t)$ and of quantity $D K(t)=\Pi / \hbar$ for the $C a+M o$ reaction is shown in Fig.1a. Here $\Pi=\frac{N Z}{A}\left(\frac{P_{p}}{Z}-\frac{P_{n}}{N}\right)$ with $P_{p}\left(P_{n}\right)$ center of mass in momentum space for protons (neutrons) is just the canonically conjugate momentum of the $X$ coordinate, $\Pi=M_{\text {coll }} X^{\prime}(t)$. Then a collective $G D R$ hamiltonian can be introduced as $H_{G D R}=$ $\Pi^{2} /\left(2 M_{\text {coll }}\right)+M_{\text {coll }} \omega_{0}^{2} X^{2} / 2$.

We choose the origin of time at the beginning of the dinuclear phase [8]. The dipole acceleration as obtained by a second order numerical derivative of $D(t)$ is shown in Fig.1b. With a good approximation this is zero before $t=0$. It suddenly rises when the collective dipole excitation is triggered and after few oscillations it becomes completely damped, around $250 \mathrm{fm} / \mathrm{c}$. The corresponding power spectrum, $\left|D^{\prime \prime}(\omega)\right|^{2}$, calculated from the Fourier transform of $D^{\prime \prime}(t)$ :

$$
D^{\prime \prime}(t)=\frac{1}{2 \pi} \int_{0}^{\infty} D^{\prime \prime}(\omega) \exp ^{-i \omega t} d \omega
$$

is plotted in Fig.1c by a dashed line.
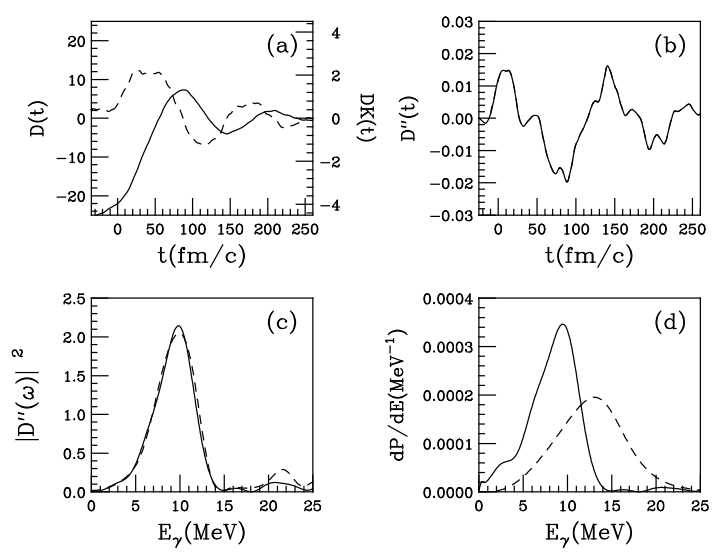

FIG. 1. System $\mathrm{Ca}+\mathrm{Mo}$ at $4 \mathrm{AMeV}$ : (a) Time evolution of $D(t)$ (solid line, in fm units), and $D K(t)$ (dashed line, in $\left.\mathrm{fm}^{-1}\right)$. (b) The same for the acceleration $D^{\prime \prime}(t)$ (in $c^{2} / f m$ ). (c) Power spectrum $\left|D^{\prime \prime}(\omega)\right|^{2}$ (in $c^{2}$ units). (d) Bremsstrahlung spectrum (solid line) and the first step statistical spectrum (dashed line).

In the same Fig.1c we have added (solid line) $\left|D^{\prime \prime}(\omega)\right|^{2}$ as obtained directly from the Fourier transform of the dipole moment, $D(\omega)$ by using:

$$
\begin{aligned}
\left|D^{\prime \prime}(\omega)\right|^{2} & =D^{\prime}(0)^{2}+\omega^{2} D(0)^{2}+2 \omega^{2} D^{\prime}(0) \operatorname{Re} D(\omega) \\
& -2 \omega^{3} D(0) \operatorname{Im} D(\omega)+\omega^{4}|D(\omega)|^{2}
\end{aligned}
$$

where $D(0)$ and $D^{\prime}(0)$ are the dipole and the dipole velocity values at $t=0$. The nice agreement between the two procedures indicates the numerical accuracy of the method.

The total photon emission probability from the preequilibrium dipole mode, given by the bremsstrahlung formula Eq.(11), is reported in Fig.1d (solid line).

For comparison we show the first step statistical spectrum (dashed line). The latter is just the product of the statistical gamma decay rate [20], (see also Eq.([)), times the mean life time of the compound nucleus $\tau_{C N}$. A good estimate of $\tau_{C N}$ can been derived from the total neutron width of a $C N$ at temperature $T: \Gamma_{n}=\hbar / \tau_{C N}=$ $2 m r_{0}^{2} A^{2 / 3} /\left(\pi \hbar^{2}\right) T^{2} \exp \left(-B_{n} / T\right)$, with $B_{n}=8.5 \mathrm{MeV}$ (neutron binding energy) and $r_{0}=1.2 \mathrm{fm}$. For the statistical $G D R$, the standard parameters from systematics, including temperature dependence of the width, are 
considered. We remark that the two contributions are comparable.

The pre-equilibrium spectrum is shifted toward lower values of energy as a consequence of the large quadrupole deformation along the fusion path. However part of the total shift is also due to the used effective interaction [6].

We perform an analogous analysis for the reaction ${ }^{16} \mathrm{O}$ $+{ }^{98} \mathrm{Mo}$ at four beam energies. In Fig.2 the power spectra $\left|D^{\prime \prime}(\omega)\right|^{2}$ obtained with the two procedures described before are plotted. Again the two methods give close results.
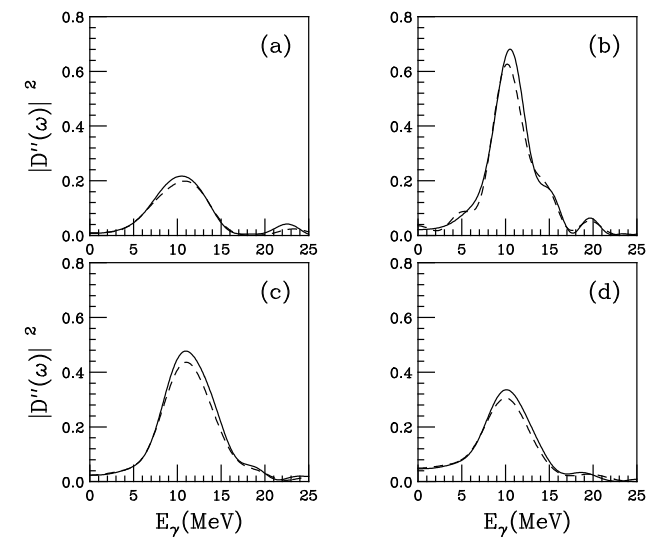

FIG. 2. Power spectrum $\left|D^{\prime \prime}(\omega)\right|^{2}$ for the system $O+M o$ at the beam energies 4 (a), 8 (b), 14 (c) and 20 (d) $A M e V$ as obtained directly from dipole acceleration (solid line) and from Eq.(7) (dashed line).

In Fig.3 the corresponding "bremss" spectra (solid lines) as well as the first step statistical spectra (dashed line) for the system $O+M o$ are plotted. We remark the characteristic "rise and fall" behaviour of the preequilibrium contribution (already observed within the "phonon" approach 8). This effect is more clearly evidenced in Fig.4 where the extra photon multiplicities due to the charge asymmetry in the entrance channel, obtained integrating over energy in the resonance region, are plotted as a function of excitation energy for both "bremss" and "phonon" (see following) models. The resulting trend is determined by the interplay of several effects. At low beam energies, a slow neck dynamics does not sustain the dipole oscillations. A strong attenuation of the acceleration takes place along entrance channel and the dipole emission is quite low. We expect a similar behaviour in deep-inelastic reactions.

At higher beam energies a faster fusion dynamics can favor the dipole oscillation. Larger amplitudes of the acceleration and more dipole oscillations enhance the preequilibrium radiation. Around 8-14 AMeV an optimum effect is attained. At higher beam energies a larger $G D R$ damping manifests and consequently the gamma emission is again reduced [8].

From a comparison between the two systems, at the same energy available in the c.m., we observe a larger pre-equilibrium emission from $\mathrm{Ca}+\mathrm{Mo}$ (black square in Fig.4). This can be related to larger values of the dipole acceleration as a result of a larger initial dipole moment amplitude.

It is interesting to compare our results to the predictions of the phonon approach, where the main input quantity is the number of $G D R$ phonons at the time of $C N$ formation. In this model a $G D R$ phonon gas is coupled to the $C N$ [5]. The phonons are decaying with a rate $\mu=\Gamma / \hbar$ and excited with a rate $\lambda$. The enhancement of $\gamma$-decay probability is given by $\Delta P_{\text {phonon }}=\left(n_{G D R}^{(0)}-\lambda / \mu\right) \gamma_{\gamma} /\left(\mu+\gamma_{e v}\right)$ were $\gamma_{\gamma}$ is the partial width for photon emission and $\gamma_{e v}$ is the evaporation decay rate. In this method one of the uncertainties is related to the estimation of the quantity $n_{G D R}^{(0)}$ which in turn requires the knowledge of the $C N$ formation time [8]. The results presented here are obtained following the usual procedures in $\vec{r}$ - and $\vec{p}$ - space to fix the thermal equilibration time, see ref. [8]. We see from Fig.4 that the values obtained in the "phonon" approach (circles) are systematically below the "bremss" evaluation, as expected from the previous discussion. In order to get the "bremss" results we need much larger values of the parameter $n_{G D R}^{(0)}$, corresponding to times well before statistical equilibrium, not consistent with the phonon model picture.
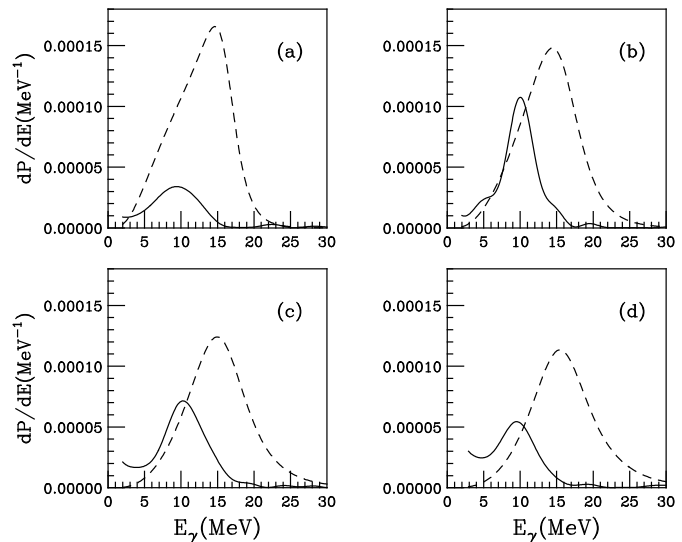

FIG. 3. Photon spectra for the $O+M o$ system at the four energies as in Fig. 2: bremsstrahlung (solid line) and first step statistical contribution (dashed line).

In Fig. 4 we report also the ratio between total preequilibrium and total statistical photon multiplicity as a function of the initial excitation energy of the compoud system. This ratio is a good measure of the preequilibrium effect which should be seen in experiments when the total photon yields from a $N / Z$ asymmetric reaction and a symmetric one, both forming the same $C N$ in similar conditions (temperature, angular momentum) are compared. To calculate the total statistical GDR contribution we consider a very schematic cascade Montecarlo model including only gamma and neutron chan- 
nel competition. The optimum range of beam energies to observe the pre-equilibrium effect appears to be around $8 \mathrm{AMeV}$. At higher beam energies a larger statistical contribution to gamma decay will lower the relative importance of pre-equilibrium contribution, which actually, as we have already discussed, starts to decrease in absolute value. We have to remark that, at the same $E^{*}$, the effect is still more important for the $\mathrm{Ca}+\mathrm{Mo}$ system, although now the total statistical emission is also larger, see Eq.(5). The $\simeq 16 \%$ enhancement is also in very good agreement with the experimental estimate of [1].
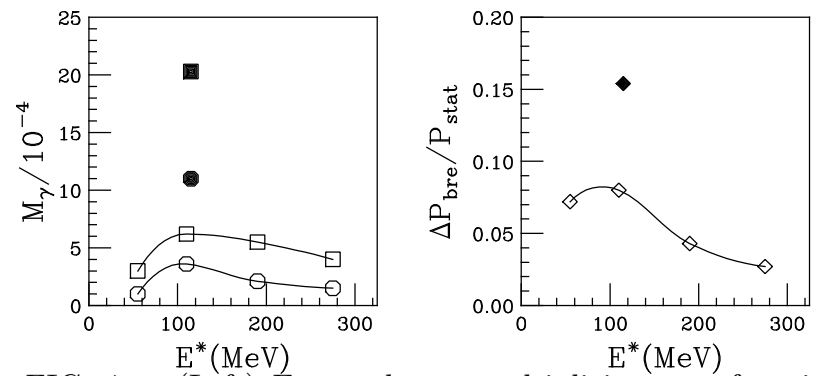

FIG. 4. (Left) Extra photon multiplicity as a function of initial excitation energy: bremsstrahlung (empty squares), phonon model (empty circles) for the $O+M o$ system and $\mathrm{Ca}+\mathrm{Mo}$ (the corresponding full symbols). (Right) Ratio of total pre-equilibrium to total statistical $G D R \gamma$-ray emission (see text).

In conclusion, in this letter we have presented a bremsstrahlung approach to dipole radiation from both thermal $G D R$ and dynamical dipole mode in fusion reactions. We stress that in our calculation we directly get absolute values for the emission probabilities without any normalization or adjusted parameters. Moreover the "bremss" approach can be extended in a similar way to dissipative deep-inelastic collisions [22] where similar prompt $\gamma-G D R$ emission has been recently observed 23 .

We have shown that when we consider the full dynamics of the pre-equilibrium dipole in charge asymmetric fusion processes the extra radiation contribution appears to be systematically two times larger than the expectations, based on a phonon model, used so far [6 8,5, 5, 11,14] (see Fig.4). The pre-equilibrium dipole radiation due to the charge asymmetry in the entrance channel can be then comparable to the statistical $C N$ emission. In this sense it can represent a new "cooling" mechanism in warm fusion reactions to form less excited residues, e.g. of interest for the synthesis of heavy elements.

A dependence of the pre-equilibrium gamma emission on beam energy has been evidenced. We conclude that this is a consequence of the interplay between fusion dynamics, damping properties of the dipole and statistical gamma emission. We expect a better observation of the effect in reactions between nuclei with a larger initial dipole amplitude, at beam energies where fusion dynam- ics is quite fast. Exotic (radioactive) beams are a good opportunity for studying these phenomena. However a caution is related to the fact that now a charge equilibration can take place also by an enhancement of fast nucleon emission.

We finally remark that the dynamical nature of the pre-equilibrium contribution will show up in a clear anisotropic $\gamma$-emission since it is due to dipole oscillations on the reaction plane (see details in [\&]). This could be a nice characteristic signature of fusion paths in reactions induced by radioactive beams.

[1] H. Hofmann et al., Z. Physik A 293229 (1979).

[2] P. Bonche, N. Ngo, Phys. Lett. B 10517 (1981).

[3] M. Di Toro, C. Gregoire, Z. Physik A 320321 (1985).

[4] E. Suraud et al., Nucl. Phys. A 492294 (1989).

[5] Ph. Chomaz et al. Nucl.Phys. A 563509 (1993).

[6] V. Baran et al., Nucl. Phys. A 600111 (1996).

[7] C. Simenel et al. Phys.Rev.Lett. 672971 (2001).

[8] V. Baran et al., Nucl. Phys. A 679373 (2001).

[9] D.M. Brink, Nucl. Phys. A 519 3c (1990).

[10] P.F. Bortignon et al. Phys.Rev.Lett. 673360 (1991).

[11] S. Flibotte et al., Phys.Rev.Lett. 771448 (1996).

[12] M. Cinausero et al., Nuovo Cimento A 111613 (1998).

[13] F. Amorini et al., Phys.Rev. C 58987 (1998).

[14] D. Pierroutsakou et al., Nucl.Phys. A 683 393c (2001).

[15] D.M.Brink, D.Phil.Thesis, Oxford 1955. P.Axel, Phys.Rev. 126671 (1962).

[16] J.D. Jackson, Classical Electrodynamics (Wiley, New York, 1962).

[17] A bremsstrahlung approach, T. Papenbrock and G.F. Bertsch Phys.Rev.Lett. 804141 (1998) was applied to calculate the photon emission during the alpha decay process. In our calculations the classical "bremss" appears justified since the dynamics is well above the threshold and so in a classical allowed region.

[18] H.B. Callen, T.A. Welton, Phys.Rev 8334 (1951).

[19] L. D. Landau and E.M. Lifshitz, Statistical Physics, (Pergamon Press, 1989), p. 386.

[20] K.A.Snover, Annu.Rev.Nucl.Part.Sci. 36545 (1986).

[21] D.M. Brink, Nucl. Phys. A 482 3c (1988).

[22] M. Papa et al. , Eur.Phys.J. A 469 (1999).

[23] M. Trotta et al., RIKEN Review 2396 (1999), M.Sandoli et al., Eur.Phys.J. A 6275 (1999). 